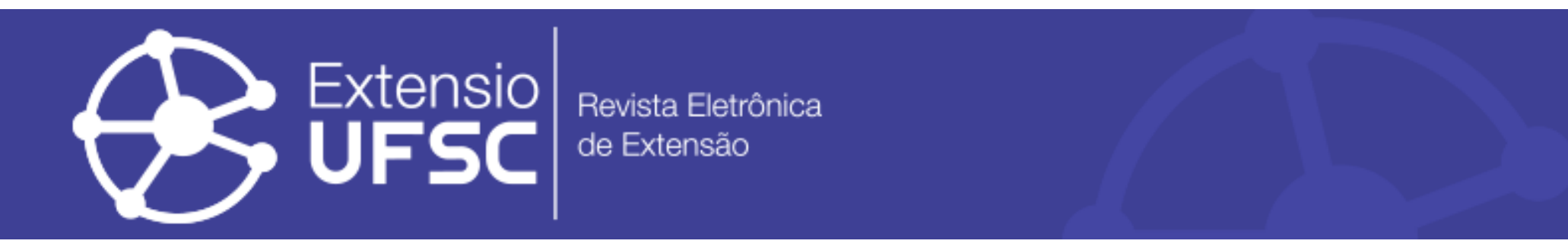

\title{
MINERAÇÃO DE DADOS APLICADA AOS RISCOS DA OBESIDADE EM CASCAVEL/ PR
}

\author{
Débora Jacomini \\ Universidade Estadual do Oeste do Paraná \\ debora.jacomini@unioeste.br \\ Eliana Mira de Bona \\ Universidade Estadual do Oeste do Paraná \\ elianamiradebona@gmail.com \\ Jerry Johann \\ Universidade Estadual do Oeste do Paraná \\ jerry.johann@hotmail.com
}

Resumo

O objetivo do estudo foi obter padrões refentes a obesidade e seus fatores de risco cardiovascular e de comorbidade na cidade de Cascavel - PR, por meio da aplicação de técnicas da Mineração de Dados (MD). Os dados sobre obesidade foram coletados durante o projeto de extensão do curso de Farmácia da Unioeste, por meio de questionário com perguntas fechadas, que foram analisadas no programa Weka, e os resultados comparados com a literatura. De acordo com as árvores de decisões geradas, os indivíduos obesos, com elevada circunferência abdominal possuem maiores riscos cardiovasculares e de comorbidades. O melhor resultado da avaliação do modelo gerado para o atributo meta-risco cardiovascular apresentou uma acurácia de 94,8\%, com 110 instâncias classificadas corretamente, e para o atributo meta-risco de comorbidade a acurácia foi de $84 \%$. A MD gerou informações úteis para a tomada de decisões a partir do banco de dados construído, auxiliando na seleção de parâmetros relevantes para a obtenção de indicadores da saúde pública.

Palavras-chave: Inteligência Artificial. Risco Cardiovascular. Risco de Comorbidade.

\section{DATA MINING APPLIED TO THE RISK OF OBESITY IN CASCAVEL/ PR}

\begin{abstract}
The aim of this study was to obtain patterns related to obesity and cardiovascular and comorbid risk factors in the city of Cascavel - PR, through the application of Data Mining (DM) techniques. Data about obesity were collected during the extension project of Unioeste's Pharmacy graduation course, by means of a questionnaire with closed questions, which were analyzed in Weka program, and the results compared to the literature. According to the generated decisions tree, the obese individuals, with high abdominal circumference, have greater cardiovascular and comorbid risks. The best result of the model generated evaluation for the cardiovascular meta-risk attribute presented an accuracy of 94.8\%, with 110 properly sorted instances, and for comorbid meta-risk attribute the accuracy was $84 \%$. The DM generated useful informations for the decisions-making through the built data bank, assisting in the relevant parameter selection to obtain indicators of public health.
\end{abstract}

Keywords: Artificial Intelligence. Cardiovascular Risk. Risk of Comorbidity.

\section{MINERÍA DE DATOS APLICADA A LOS RIESGOS DE LA OBESIDAD EN CASCAVEL/ PR}

\section{Resumen}

El objetivo obtener patrones refentes a la obesidad y sus factores de riesgo cardiovascular y de comorbilidad en la ciudad de Cascavel - PR, por medio de la aplicación de técnicas de la minería de datos (MD). Los datos sobre obesidad fueron recolectados durante el proyecto de extensión del curso de Farmacia de la Unioeste, por medio de un cuestionario con preguntas cerradas, que fueron analizadas en el programa Weka, y los resultados comparados con la literatura. De acuerdo con los árboles de decisiones generadas, los individuos obesos, con elevada circunferencia abdominal poseen mayores riesgos cardiovasculares y de comorbilidades. El mejor resultado de la evaluación del modelo generado para el atributo meta-riesgo cardiovascular presentó una exactitud del 94,8\%, con 110 instancias clasificadas correctamente, y para el atributo meta-riesgo de comorbilidad la exactitud fue del $84 \%$. La MD generó información útil para la toma de decisiones a partir de la base de datos construida, ayudando en la selección de parámetros relevantes para la obtención de indicadores de salud pública.

Palabras clave: Inteligencia Artificial. Riesgo Cardiovascular. Riesgo de Comorbilidad. 


\section{INTRODUÇÃO}

Obesidade e sobrepeso são definidos pela Organização Mundial da Saúde (OMS) como o acúmulo excessivo de gordura e aumento de peso corporal, que pode prejudicar a saúde do ser humano (WHO, 2015). Esta desordem, provavelmente, é o mais antigo distúrbio metabólico do mundo, havendo relatos de sua ocorrência em múmias egípcias e em esculturas gregas (FRANCISCHI et al., 2000). Ao longo da história da humanidade, ganho de peso e depósitos exagerados de gordura foram vistos como sinais de saúde e prosperidade (REPETTO; RIZZOLLI; BONATTO, 2003). Hoje, no entanto, a obesidade se torna uma ameaça a saúde da população, devido à transição nos padrões nutricionais, relacionados às mudanças demográficas, socioeconômicas e epidemiológicas do último século (TEICHMANN et al., 2006).

A prevalência de obesidade tem aumentado em todo o mundo e vem se tornando o maior problema de saúde na sociedade moderna, na maioria dos países desenvolvidos e em alguns subdesenvolvidos (WHO, 2000). Em 2013, a proporção de adultos obesos no mundo subiu de 28,8 para 36,9\% entre homens, e de 29,8 para 38\% entre as mulheres (WHO, 2015). E nas últimas décadas a alta prevalência de obesidade na população mundial, foi responsável por 2,8 milhões de mortes por ano (REIS et al., 2017).

No Brasil, dados divulgados da pesquisa de orçamentos familiares, realizada pelo Instituto Brasileiro de Geografia e Estatística (IBGE, 2010) revela um aumento no sobrepeso e obesidade da população, atingindo 49\% da população no país. A Vigilância de Fatores de Risco e Proteção para Doenças Crônicas por Inquérito Telefônico (VIGITEL) do Ministério da Saúde (MS), também obteve dados similares em seu estudo, no qual 51\% da população brasileira está acima do peso ideal (BRASIL, 2014). Dentre as regiões do País, o Sul apresenta as maiores prevalências de obesidade, sendo essas semelhantes ou superiores as obtidas em países desenvolvidos (GIGANTE et al., 1997). Isso ocorre, segundo a Sociedade Brasileira de Cardiologia (SBC) devido ao sedentarismo de $80 \%$ da população, o que acarreta no desenvolvimento de obesidade em 32\% dos casos (SOCIEDADE BRASILEIRA DE CARDIOLOGIA, 2002).

A obesidade é uma doença grave, multifacetada e de genética complexa, com importantes implicações sociais, psicológicas e médicas (SOUZA et al., 2003). As consequências do excesso de peso à saúde associam-se, com grande frequência, a doenças crônicas como hipertensão arterial, diabetes mellitus (CANTOS; SOUZA, 2012), hipercolesterolemia, aterosclerose, doenças cardiovasculares, neoplasias, apneia do sono, gota, cálculo urinário, distúrbios psicossociais e osteoartrites (TEICHMANN et al., 2006). Reis (2017) afirma em seu estudo, que a obesidade está associada principalmente à quadros de hipertensão. 
A obesidade, além de acompanhar altos índices de comorbidade (associação de doenças), também é responsável por elevadas taxas de mortalidade, principalmente, decorrentes de doenças coronarianas. A associação entre obesidade e doenças cardíacas ocorre, principalmente, em grupos de obesos que apresentam adiposidade localizada na região abdominal ou central, mesmo na ausência da obesidade generalizada (PITANGA; LESSA, 2005).

O diagnóstico correto da obesidade busca a identificação de níveis de risco, o que requer algumas formas de quantificação. A medida mais utilizada é o índice de massa corporal [IMC $\left(\mathrm{Kg} / \mathrm{m}^{2}\right)=$ Peso $(\mathrm{Kg}) /$ Altura $\left.(\mathrm{m})^{2}\right]$, que consiste em uma razão entre peso e altura (WHO, 2000). Porém, este cálculo apresenta algumas limitações, pois não distingue massa gordurosa de massa magra, sendo difícil estimar em indivíduos mais velhos, em decorrência de sua perda de massa magra e diminuição do peso, e superestimado em indivíduos musculosos. Também, não reflete, necessariamente, a distribuição de gordura corporal (ABESO, 2009).

Desta forma, para o diagnóstico correto de obesidade, é necessário combinar o cálculo do IMC com outra avaliação da distribuição de gordura corporal, a medida da circunferência abdominal (CA) é a mais utilizada. A CA reflete melhor o conteúdo de gordura visceral e possui alta relação com a gordura corporal total. A junção dessas medidas oferece uma forma combinada de avaliação de risco e ajuda a diminuir as limitações de cada um dos métodos (ABESO, 2009).

Os dados sobre obesidade são diversos e difíceis de serem associados, por isso técnicas como a Descoberta de Conhecimento em Bases de Dados (Knowledge Discovery in Database KDD) mostram-se efetivas na gestão dessas informações. A Mineração de Dados (Data Mining DM) é um dos processos do KDD, que utiliza métodos e técnicas que permitem analisar grandes conjuntos de dados para extração de informação desconhecida e válida, que geram ações úteis, de grande ajuda para a tomada de decisões estratégicas (BUENO; VIANA, 2012). É uma área emergente dentro da inteligência artificial usada na análise de grandes bancos de dados, com a geração de padrões e extração de informações relevantes (VIANNA et al., 2010). A MD foi originalmente desenvolvida com objetivos comerciais, mas tem sido amplamente adotada por outras áreas, inclusive a da saúde (CARVALHO et al., 2012).

Uma das tarefas da MD é a classificação que permite obter padrões a partir da construção de classificadores, como as árvores de decisões, que manipulam tanto atributos nominais como quantitativos. As árvores de decisões são largamente utilizadas, devido à sua eficiência em relação ao tempo de processamento, e por fornecer um meio intuitivo de analisar os resultados obtidos, apresentando uma forma de representação simbólica, simples e normalmente compreensível, o que facilita a análise do problema de qualquer área de interesse (GARCIA, 2003). 
Mineração de dados aplicada aos riscos da obesidade em Cascavel/PR

Sendo assim, o objetivo desse estudo foi obter padrões refente a obesidade, por meio da análise de seus fatores de risco cardiovascular e risco de comorbidade na cidade de Cascavel PR, utizando técnicas de mineração de dados. Este trabalho explora, mediante a realização de estudo de caso, o uso de árvores de decisão que realiza aprendizado não supervisionado, para avaliar a adequação desta tecnologia em uma base de dados real da área de saúde.

\section{MATERIAIS E MÉTODOS}

\section{Coleta de dados}

Os dados foram coletados em agosto de 2015, durante o projeto de extensão promovido pelo Curso de Farmácia da Universidade Estadual do Oeste do Paraná - UNIOESTE. Dentre suas atividades, o projeto promove anualmente uma campanha de uso racional de medicamentos na praça central do município de Cascavel - PR. O objetivo da campanha é ofertar vários serviços à população, incluindo a determinação do IMC e da CA.

No momento do atendimento ao paciente, um questionário com dez perguntas fechadas foi aplicado, visando um estudo sobre a obesidade na população de Cascavel. As classificações do IMC e da CA foram feitas segundo as recomendações da OMS, Quadro 1 e 2, respectivamente.

Quadro 1. Classificação de peso pelo IMC e avaliação do risco de comorbidade

\begin{tabular}{lll}
\hline Classificação & IMC $\left(\mathbf{K g} / \mathbf{m}^{2}\right)$ & Risco Comorbidade \\
\hline Baixo Peso & $<18,5$ & Baixo \\
Peso Normal & 18,5 a 24,9 & Médio \\
Sobrepeso & 25,0 & Médio Aumentado \\
Pré-obeso & 25,1 a 29,9 & Médio Aumentado \\
Obeso I & 30,0 a 34,9 & Moderado \\
Obeso II & 35 a 39,9 & Grave \\
Obeso III & $\geq 40,0$ & Muito Grave \\
\hline
\end{tabular}

Quadro 2. CA e risco cardiovascular associada a obesidade em homens e mulheres.

\begin{tabular}{lll}
\hline \multicolumn{2}{l}{ Circunferência Abdominal $(\mathrm{cm})$} & $\begin{array}{l}\text { Risco } \\
\text { Cardiovascular }\end{array}$ \\
\cline { 1 - 2 } Homem & Mulher & \\
\hline$<94$ & $<80$ & Baixo
\end{tabular}




\begin{tabular}{lll}
$\geq 94$ & $\geq 80$ & Médio \\
$\geq 102$ & $\geq 88$ & Grave \\
\hline
\end{tabular}

\section{Processo KDD}

O processo de KDD foi constituído por cinco etapas: seleção, pré-processamento, transformação, mineração dos dados e interpretação do resultado (Figura 1).

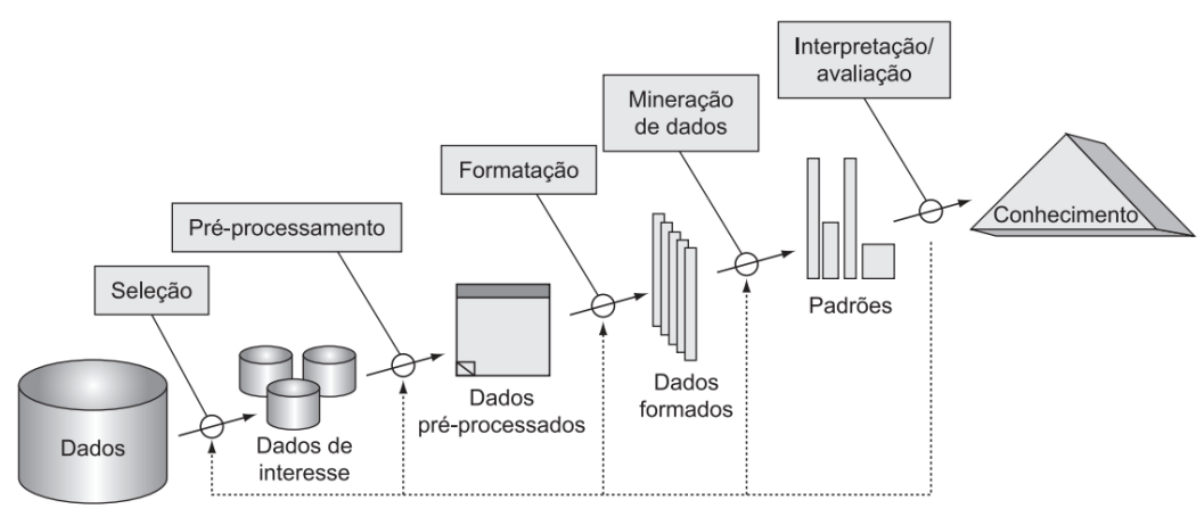

Figura 1. Etapas do Processo KDD

Fonte: (SILVA NETO et al., 2010).

\section{Seleção de Dados}

A seleção dos dados é a primeira fase do KDD, na qual foi decidido o conjunto de dados que forneceria conhecimentos úteis. O conjunto de dados foi obtido após a tabulação das informações coletadas na campanha. O banco de dados gerado possuía 9 atributos: sexo, peso, altura, IMC, CA, número de refeições por dia, frequência de exercício físico por semana, risco cardiovascular e risco de comorbidade. A base de dados possuía um total de 116 instâncias, na qual cada instância corresponde a um paciente. Não houve valores faltantes, pois, os pacientes atendidos responderam a todas as perguntas do questionário.

\section{Pré-processamento de Dados}

A fase de pré-processamento compreendeu a categorização de alguns atributos. Neste banco de dados sobre a obesidade houve a necessidade de categorizar apenas dois atributos: o risco cardiovascular, que foi dividido em 3 categorias (baixo, médio e grave), e o risco de comorbidade em 6 categorias (baixo, médio, médio/aumentado, moderado, grave e muito grave). Esta preparação dos atributos foi estabelecida conforme a classificação recomendada pela Diretriz Brasileira de Obesidade (ABESO, 2009). Os dados foram salvos no formato separados 
Mineração de dados aplicada aos riscos da obesidade em Cascavel/PR

por vírgula (csv), para posterior análise em software Weka (Waikato Environment for Knowledge Analysis) (UNIVERSITY OF WAIKATO, 2010).

\section{Transformação dos dados}

A transformação consiste na organização dos dados de forma específica, para que a próxima etapa do KDD possa ser realizada. Assim, os dados tabelados foram transportados para o Weka, para a classificação dos atributos.

\section{Mineração de Dados}

$\mathrm{Na}$ etapa de MD são extraídas regras que permitem relacionar as diversas variáveis sob estudo. Para sua realização, utilizou-se o algoritmo J48, constituído uma versão do algoritmo C4.5, que representa as relações entre as variáveis no formato de uma árvore de decisão, a partir da qual podem ser lidas as regras.

Uma árvore de decisão consiste em um conjunto hierarquicamente ordenado de conceitos, que iniciam em um nó raiz, no qual cada conceito é dividido em sub-conceitos no próximo nível da árvore. Cada elemento terminal de uma árvore (conhecido como nó folha) está associado a um conceito objetivo (VIANNA et al., 2010).

Como o processo de MD é interativo, foram realizados vários experimentos. A tarefa de classificação aplicada neste trabalho relacionou cada instância de triagem com os riscos à saúde, com base nos demais atributos preditivos, computacionalmente. $\mathrm{O}$ algoritmo de árvore de decisão J48 foi aplicado com os parâmetros: número mínimo de instâncias por nó igual a 2 e fator de confiança mínima utilizada em podas igual a 0,25 . O experimento foi configurado para testar o modelo obtido com uma abordagem de validação cruzada com 12 partições para o atributo risco cardíaco e com 9 partições para o risco de comorbidade. Neste último atributo também foi aplicado o método Pruning para a realização de poda.

\section{RESULTADOS E ANÁLISES}

Os atributos foram classificados por meio da estatística descritiva. A partir destes dados geraram-se duas árvores de decisões com suas regras (R) de classificação, considerando como atributos meta o nível de risco cardíaco (Figura 2) e risco de comorbidade (Figura 3). Estes atributos metas foram escolhidos para determinar quais os riscos da obesidade para a população de Cascavel - PR. 
A acurácia de um modelo é considerada como o principal indicador de sucesso da classificação (MACIEL et al., 2015). Por isso, considerou-se para a discussão as regras com o maior número de instâncias corretamente classificadas e com acurácia maior ou igual a 80\%.

O melhor resultado da avaliação do modelo gerado para o atributo meta risco cardiovascular apresentou uma acurácia de 94,8\%, com 110 instâncias classificadas corretamente.

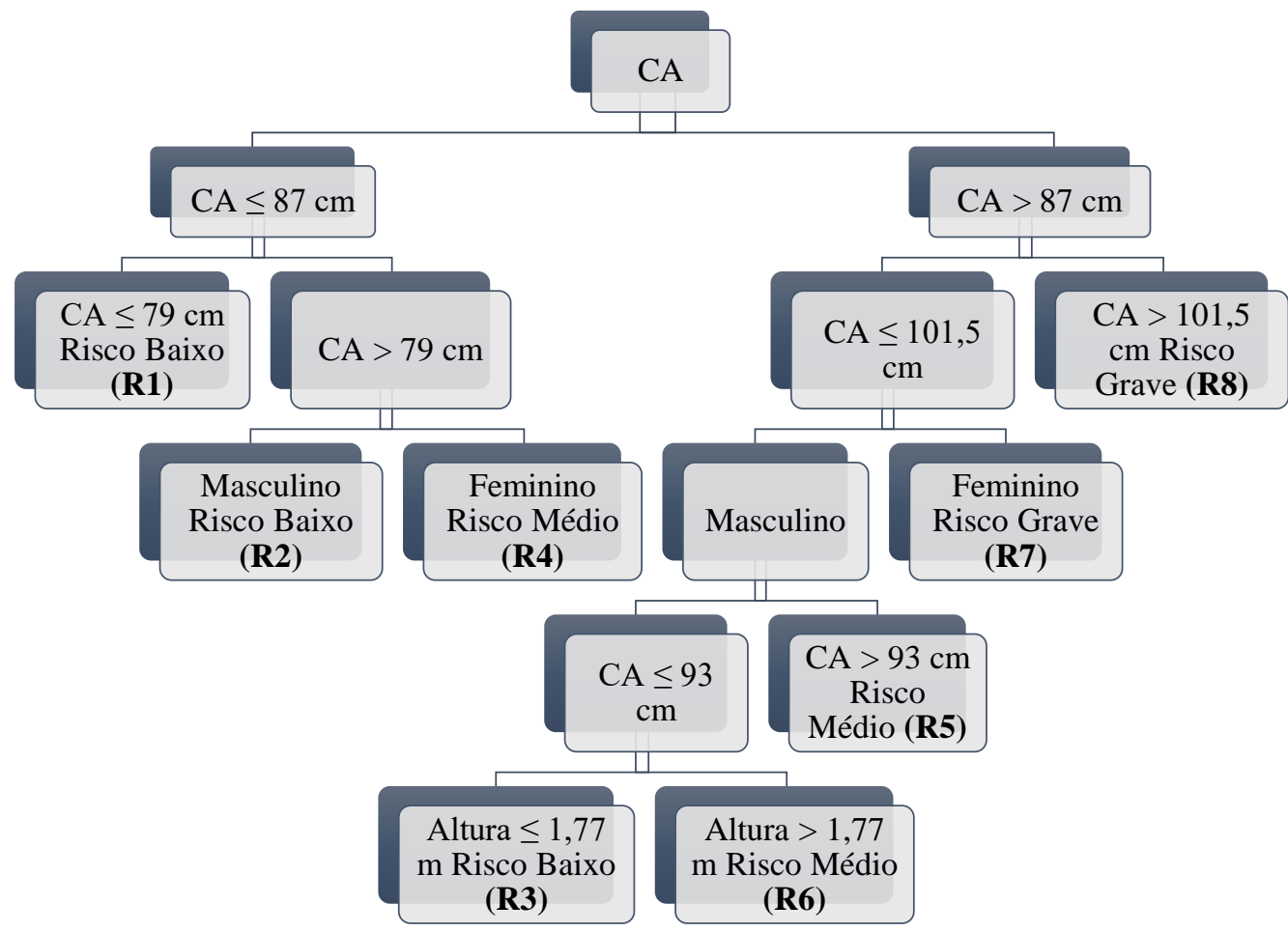

\begin{tabular}{|c|c|}
\hline Regras de Classificação & Descrição \\
\hline R1 & Se CA $\leq 79 \mathrm{~cm}$, então o risco cardiovascular será Baixo \\
\hline $\mathbf{R} 2$ & $\begin{array}{l}\text { Se CA }>79 \mathrm{~cm} \text { e o paciente for do sexo Masculino, então o risco cardiovascular será } \\
\text { Baixo }\end{array}$ \\
\hline R3 & $\begin{array}{l}\text { Se } 87<\text { CA } \leq 93 \mathrm{~cm} \text { e o paciente for do sexo masculino com altura } \leq 1,77 \mathrm{~cm} \text {, então } \\
\text { o risco cardiovascular será Baixo }\end{array}$ \\
\hline R4 & $\begin{array}{l}\text { Se CA }>79 \mathrm{~cm} \text { e o paciente for do sexo Feminino, então o risco cardiovascular será } \\
\text { Médio }\end{array}$ \\
\hline R5 & $\begin{array}{l}\text { Se } 93<\text { CA } \leq 101,5 \mathrm{~cm} \text { e o paciente for do sexo Masculino, então o risco } \\
\text { cardiovascular será Médio }\end{array}$ \\
\hline R6 & $\begin{array}{l}\text { Se } 87<\text { CA } \leq 93 \mathrm{~cm} \text { e o paciente for do sexo Masculino com altura }>1,77 \mathrm{~cm} \text {, então } \\
\text { o risco cardiovascular será Médio }\end{array}$ \\
\hline R7 & $\begin{array}{l}\text { Se } 87<\text { CA } \leq 101,5 \mathrm{~cm} \text { e o paciente for do sexo Feminino, então o risco } \\
\text { cardiovascular será Grave }\end{array}$ \\
\hline R8 & Se CA > 101,5 cm, então o risco cardiovascular será Grave \\
\hline
\end{tabular}

Figura 2. Árvore e regras de decisão com base no atributo meta risco cardiovascular. Fonte: Elaborado pelos autores. 
Segundo a matriz de confusão gerada para o atributo risco cardiovascular, mais de 50\% dos entrevistados foram classificados com risco cardíaco grave, de acordo com dados da OMS (WHO, 2000). Resultado semelhante foi obtido por Souza et al. (2003), em triagem no Rio de Janeiro - RJ em 2003, quando entrevistaram 1039 adultos por meio de questionário fechado, e concluiram que destes 57,7\% apresentaram algum problema cardíaco.

Ao comparar as regras de decisão R2 e R4, que são provenientes do mesmo nó interno da árvore da Figura 2, que classificam os casos em que a CA é maior que $79 \mathrm{~cm}$, observa-se que o risco cardiovascular está relacionado principalmente ao sexo feminino. Essa regra se repete nas folhas classificadas como R3, R5, R6 e R7, que também são proveniente de um mesmo nó interno, em que a CA é menor ou igual a $101,5 \mathrm{~cm}$, nestes ramos apenas o sexo feminino (R7) apresenta risco grave. O maior perigo cardíaco apresentado pelos homens foi o risco médio, isso quando a CA for maior que $93 \mathrm{~cm}$ e/ ou a altura for maior que $1,77 \mathrm{~m}$, respectivamente as regras R5 e R6.

A porcentagem de casos com risco cardíaco grave para o sexo feminino, contabilizados no banco de dados de Cascavel - PR, correspondeu a mais de 65\% do total de mulheres analisadas. Em uma pesquisa sobre obesidade, realizada em Campinhas - SP no ano 2000, as mulheres também foram descritas como o grupo de maior risco coronariano, apresentando um risco duas vezes mais elevado de desenvolver doenças cardíacas quando comparadas aos homens, sendo (FRANCISCHI et al., 2000), dados que coroboram com as regras de decisão da Figura 2.

Observa-se também, que independente do sexo ou de qualquer outro atributo, a CA esta relacionada diretamente ao risco cardíaco. Sendo que, pessoas com CA menor que $79 \mathrm{~cm}$ apresentam risco cardíaco baixo (R1) e, pacientes com CA maior que 101,5 cm apresentam risco cardíaco grave (R8). Apesar das doenças coronarianas representarem a maior causa de mortes relacionadas ao excesso de peso, as pessoas obesas também desenvolvem outras comorbidades que as predispõem à mortalidade: diabetes mellitus, dislipidemias, hipertensão, doenças cardiovasculares, doenças do trato digestivo, neoplasias, disfunções endócrinas, disfunção da vesícula biliar, problemas pulmonares, artrites, entre outras (SOUZA et al., 2003).

Considerando esta relevância do risco de comorbidade, também foi gerada uma árvore de decisão considerando-o como atributo meta (Figuras 3). A acurácia do modelo para este atributo foi de 84\%, com 98 instâncias classificadas corretamente. 


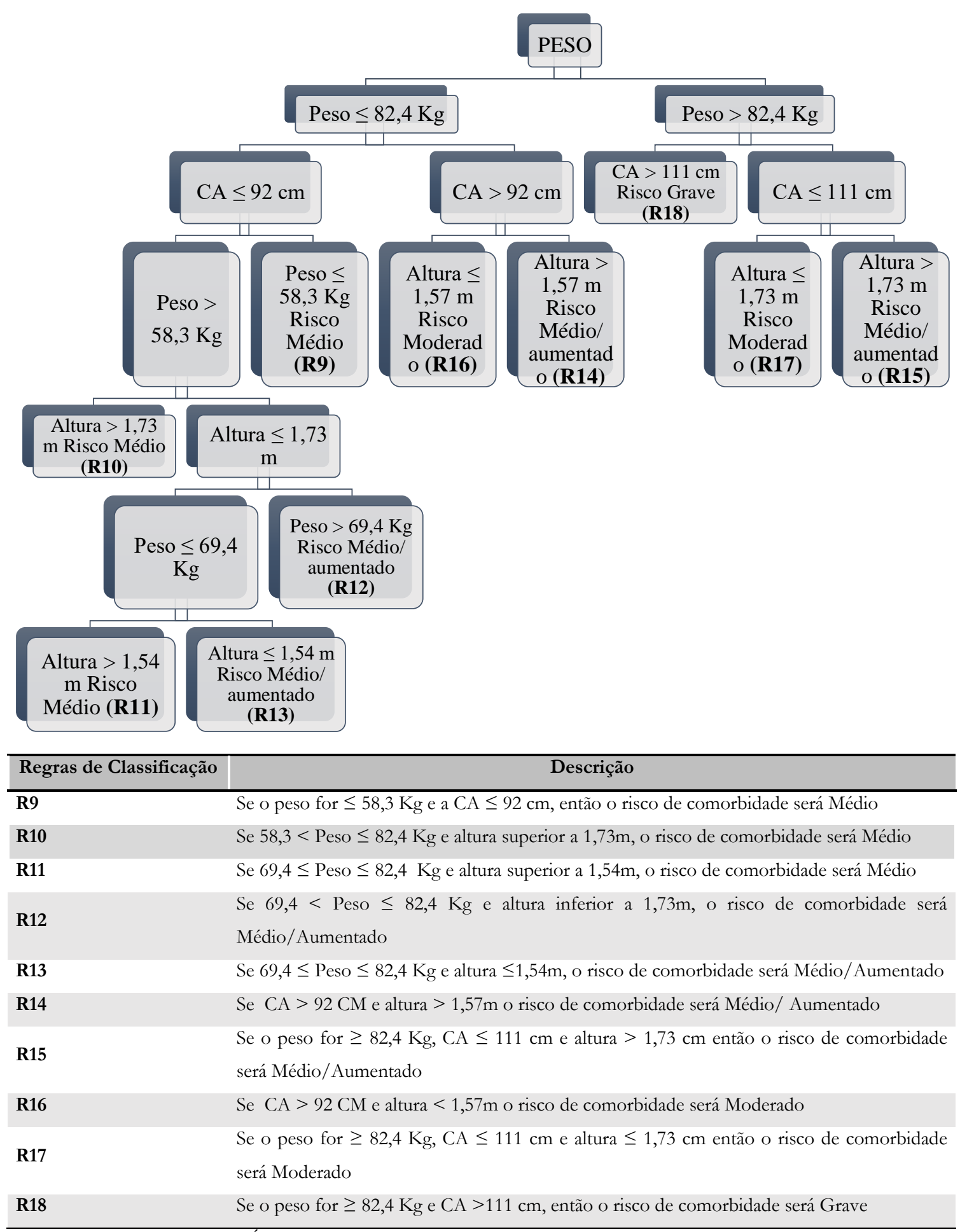

Figura 3. Árvore e regras de decisão com base no atributo meta risco de comorbidade. Fonte: Elaborado pelos autores.

De forma geral, o risco de comorbidade esta relacionado diretamente ao peso, CA e altura do paciente. Pacientes com menores CA $(\leq 92 \mathrm{~cm})$, peso $(\leq 58,3 \mathrm{Kg})$ e maiores alturas $(>1,73$ m) apresentaram risco médio de comorbidade, respectivamente classificados nas regras R11, R9 e R10. Por outro lado, os pacientes com maiores CA e peso, possuem risco grave de comorbidade (R18). Para valores intermediários desses parâmetros a classificação permaneceu entre o risco 
médio/aumentado e moderado, R12 a R17. Nenhum paciente foi classificado, quanto ao risco de comorbidade, em baixo ou muito grave. Valores encontrados para circunferência abdominal, acima de $111 \mathrm{~cm}$, estão relacionados ao maior risco de comorbidade (grave).

$\mathrm{O}$ risco de comorbidade também pode ser associado a maiores $\mathrm{CA}$ em indivíduos com menor estatura e maior peso. Segundo a literatura aumenta-se o risco de desenvolver comorbidades, ao aumentar-se o peso corporal. As complicações da obesidade não dependem apenas do excesso de peso, mas também da distribuição da gordura corporal, uma vez que a presença de tecido adiposo intra-abdominal também é um fator de risco para distúrbios metabólicos. Sendo assim, quanto maior a CA e o peso corporal, maior será o risco de desenvolver várias doenças simultaneamente (GRUNDY, 1998).

Os maiores riscos a saúde causados pela obesidade aumentam progressivamente e desproporcionalmente com o aumento de peso. Comparando obesos com pessoas de peso normal, homens 20\% acima do peso desejável tem 20\% a mais de chance de morrer por decorrência de alguma comorbidade. Ainda, possuem o risco duas vezes maior de falecer por diabetes, tem $40 \%$ a mais de chance de desenvolver disfunções na vesícula biliar e $25 \%$ a mais de predisposição à doenças coronarianas. Em homens com 40\% acima do peso desejável, a mortalidade por diversas patologias é 55\% maior, apresentam $70 \%$ a mais de chance de desenvolver doenças coronarianas, e o risco de morte por diabetes é quatro vezes maior do que entre pessoas de peso normal (FRANCISCHI et al., 2000).

Os números de refeições por dia e exercícios físicos não foram classificados em nenhuma árvore de decisão. Porém, Segundo Gigante e colaboradores (1997) eles são considerados importantes marcadores de risco, uma vez que o consumo concentrado de calorias, em uma única refeição diária, está associado à formação rápida de gordura, e seu depósito na região abdominal, aumentando de forma indireta o risco cardíaco e de comorbidades (GIGANTE et al., 1997). Já a prática de atividade física pode influenciar de maneira positiva a composição corporal por meio de vários mecanismos, pois leva ao aumento do gasto energético total, promovendo um equilíbrio na oxidação dos macronutrientes e preservação da massa magra (MONTEIRO et al., 2004).

Verificou-se pelos dados obtidos que o risco de comorbidade e os níveis de risco cardíaco não se relacionam diretamente. Contudo, os atributos relacionados a eles, em suas respectivas árvores de decisões e nas regras geradas, correspondem aos dados encontrados na literatura, como a relação do IMC com o atributo risco de comorbidade, e da CA com o atributo risco cardíaco. Vale ressaltar que, em outras áreas da saúde, a utilização do KDD também permitiu agregar significativo poder de decisão, como no trabalho de Malucelli e colaboradores (2010) no 
qual classificaram microáreas na cidade de Curitiba - PR, para facilitar o trabalho das equipes de saúde. Na pesquisa de Vianna e colaboradores (2010), que definiram regras importantes relacionadas a mortalidade infantil. No artigo de Carvalho et al. (2012) que analisaram o prontuário de vários pacientes por $\mathrm{MD}$, e perceberam que desta forma podem aproveitar melhor o potencial dos dados disponíveis, gerando regras úteis para a prática clínica.

\section{CONSIDERAÇÕES FINAIS}

Este trabalho demonstra que é possível utilizar as técnicas de MD em Saúde Pública, obtendo-se conclusões relevantes. Como a regra de que indivíduos obesos, principalmente aqueles com excesso de adiposidade no abdômen, apresentam em relação à população normal uma associação maior a fatores de risco cardiovascular e de comorbidades. Considerando que o algoritmo foi satisfatório em termos de precisão, as regras simples geradas permitirão classificar riscos a saúde de novos indivíduos na prática, sem a necessidade que novas análises sejam realizadas. Essa ferramenta poderá auxiliar na triagem de pacientes e garantir confiança nas predições de risco. Sendo assim, conclui-se que os devidos registros e armazenamentos destes dados coletados ao longo do projeto podem contribuir não apenas com a composição de valioso banco de dados, mas, principalmente, com o uso desses dados, visando a minerar o que está subjacente aos dados brutos e trazer à tona informações que levarão a descobertas importante para o redirecionamento de práticas e a tomada de decisões.

\section{REFERÊNCIAS}

ABESO. Diretriz Brasileiras de Obesidade. Acta Farmacêutica, v. 3, p. 85, 2009.

BRASIL. VIGITEL Brasil 2013. Brasil. Ministério da Saúde. Secretaria de Vigilância em Saúde., p. 120p, 2014.

BUENO, M. F.; VIANA, M. R. Mineração de dados: Aplicações, Eficiência e Usabilidade. Anais do congresso de iniciaşão científica do INATEL - INCITEL. 2012

CANTOS, G. A.; SOUZA, L. C. Acompanhamento de dois pacientes diabéticos por equipe interdisciplinar e multiprofissional. Extensio, v. 9, n. 14, p. 81-91, 2012.

CARVALHO, D. R. et al. Mineração de Dados aplicada à fisioterapia. Fisioter. Mov., v. 25, n. 3, p. 595-605, 2012.

FRANCISCHI, R. P. P. DE et al. Obesidade: atualização sobre sua etiologia, morbidade e tratamento. Revista de Nutrição, v. 13, n. 1, p. 17-28, 2000. 
GARCIA, S. C. O Uso de Árvores de Decisão na Descoberta de Conbecimento na Área da Saúde. 2003. 88 f. 2003. Disponível em: < http://hdl.handle.net/10183/19032>.

GIGANTE, D. P. et al. Prevalência de obesidade em adultos e seus fatores de risco. Revista de Saúde Publica, v. 31, n. 3, p. 236-246, 1997.

GRUNDY, S. M. Multifactorial causation of obesity: implications for prevention. American journal of clinical nutrition, v. 67, n. 3, p. 540-449, 1998.

IBGE. Pesquisa de Orçamentos Familiares 2008-2009. 2010.

MACIEL, E. DA S. et al. Atributos de qualidade do pescado relacionados ao consumo na cidade de Corumbá, MS. Boletim do Instituto de Pesca, v. 41, n. 1, p. 199-206, 2015.

MALUCELLI, A. et al. Classificação de microáreas de risco com uso de mineração de dados. Revista Saúde Pública, v. 44, n. 2, p. 292-300, 2010.

MONTEIRO, N. et al. Efeitos de um programa de atividade física regular sobre os níveis séricos basais de IGF-1 em idosas. Fitness \& Performance Journal, v. 3, n. 3, p. 130-135, 2004.

PITANGA, F. J. G.; LESSA, I. Indicadores Antropométricos de Obesidade como Instrumento de Triagem para Risco Coronariano Elevado em Adultos na Cidade de Salvador - Bahia. Arquivos Brasileiros de Cardiologia, v. 85, n. 1, p. 26-31, 2005. Disponível em: <http://www.ncbi.nlm.nih.gov/pubmed/20412314>.

REIS, L. A. P. DOS et al. Obesity, hypertension and diabetes among truck drivers in the middlewest, Brazil. Biosci. J. Uberlândia, v. 33, n. 2, p. 485-493, 2017.

REPETTO, G.; RIZZOLLI, J.; BONATTO, C. Prevalência, riscos e soluções na obesidade e sobrepeso: Here, There, and Everywhere. Arquivos Brasileiros de Endocrinologia \& Metabologia, v. 47, p. 633-635, 2003. Disponível em: <http://www.scielo.br/pdf/abem/v47n6/a01v47n6.pdf>.

SILVA NETO, M. A. et al. Técnicas de mineração visual de dados aplicadas aos dados de instrumentação da barragem de Itaipu. Gestão \& Produção, v. 17, n. 4, p. 721-734, 2010. Disponível em: $\quad<$ http://www.scielo.br/scielo.php?script=sci_arttext\&pid=S0104530X2010000400007\&lng=pt\&nrm=iso\&tlng $=$ en $>$.

SOCIEDADE BRASILEIRA DE CARDIOLOGIA. Diretrizes para Avaliação e Tratamento de Pacientes com Arritmias Cardíacas. Arquivo Brasileiro de Cardiologia, v. 79, n. suplemento V, p. 150, 2002.

SOUZA, L. J. DE et al. Prevalência de obesidade e fatores de risco cardiovascular em Campos, Rio de Janeiro. Arquivos Brasileiros de Endocrinologia \& Metabologia, v. 47, n. 6, p. 669-676, 2003.

TEICHMANN, L. et al. Fatores de risco associados ao sobrepeso e a obesidade em mulheres de São Leopoldo, RS. Revista Brasileira de Epidemiologia, v. 9, n. 3, p. 360-373, 2006. Disponível em: $<$ http://www.scielo.br/scielo.php?script=sci_arttext\&pid=S1415-

790X2006000300010\&lng=pt\&nrm=iso\&tlng $=$ pt $>$. 
Mineração de dados aplicada aos riscos da obesidade em Cascavel/PR

VIANNA, R. C. X. F. et al. Mineração de dados e características da mortalidade infantil. Cadernos de Saúde Pública. , 2010

WHO. Media centre Obesity and overweight. Disponível em: $<$ www.who.int/mediacentre/factsheets/fs311/en/>.

WHO. Obesity: preventing and managing the global epidemic. . Geneva: 2000.

Recebido em: 17/07/2017

Aceito em: 02/05/2018 